\title{
Smartphone viewing distance and sleep: an experimental study utilizing motion capture technology
}

\author{
Michitaka Yoshimura ${ }^{1, *}$ \\ Momoko Kitazawa ${ }^{1-3, *}$ \\ Yasuhiro Maeda ${ }^{2}$ \\ Masaru Mimura ${ }^{4}$ \\ Kazuo Tsubota' \\ Taishiro Kishimoto ${ }^{4,5}$ \\ 'Department of Ophthalmology, \\ Keio University School of \\ Medicine, Tokyo, ${ }^{2}$ RIKEN Center \\ for Advanced Photonics, Wako, \\ Saitama, ${ }^{3}$ Department of Nursing, \\ Aino University Junior College, \\ ${ }^{4}$ Department of Neuropsychiatry, \\ Keio University School of Medicine, \\ Tokyo, Japan; ${ }^{5}$ Department of \\ Psychiatry, Hofstra Northwell School \\ of Medicine, NY, USA \\ *These authors contributed equally to \\ this work
}

This article was published in the following Dove Press journal:

Nature and Science of Sleep

8 March 2017

Number of times this article has been viewed

\begin{abstract}
There are studies reporting the negative impact of smartphone utilization on sleep. It is considered that reduction of melatonin secretion under the blue light exposure from smartphone displays is one of the causes. The viewing distance may cause sleep disturbance, because the viewing distance determines the screen illuminance and/or asthenopia. However, to date, there has been no study closely investigating the impact of viewing distance on sleep; therefore, we sought to determine the relationship between smartphone viewing distance and subjective sleep status. Twenty-three nursing students (mean age \pm standard deviation of 19.7 \pm 3.1 years) participated in the study. Subjective sleep status was assessed using the Pittsburgh Sleep Quality Index, morningness-eveningness questionnaire, and the Epworth sleepiness scale. We used the distance between the head and the hand while holding a smartphone to measure the viewing distance while using smartphones in sitting and lying positions. The distance was calculated using the three-dimensional coordinates obtained by a noncontact motion-sensing device. The viewing distance of smartphones in the sitting position ranged from 13.3 to $32.9 \mathrm{~cm}$ among participants. In the lying position, it ranged from 9.9 to $21.3 \mathrm{~cm}$. The viewing distance was longer in the sitting position than in the lying position (mean \pm standard deviation: $20.3 \pm 4.7 \mathrm{vs}$ $16.4 \pm 2.7$, respectively, $P<0.01)$. We found that the short viewing distance in the lying position had a positive correlation to a poorer sleep state $\left(R^{2}=0.27, P<0.05\right)$, lower sleep efficiency $\left(R^{2}=0.35\right.$, $P<0.05)$, and longer sleep latency $\left(R^{2}=0.38, P<0.05\right)$. Moreover, smartphone viewing distances in lying position correlated negatively with subjective sleep status. Therefore, when recommending ideal smartphone use in lying position, one should take into account the viewing distances.
\end{abstract}

Keywords: smartphone, sleep, blue light, distance

\section{Introduction}

With the dramatic progress of technology and the growing number of software developments, mobile devices have become thoroughly integrated into our daily lives. Smartphones are used not only for telecommunications but also for various leisure activities, such as net surfing, social networking, games, and taking photos/videos. Due to the reliance on and heavy usage of such devices, people's lifestyles have greatly changed.

Besides the positive aspects of such devices, we need to take into consideration the negative aspects of their use. A growing amount of evidence suggests that there are potential negative impacts from smartphones on biophysiological processes, especially on sleep. Munezawa et $\mathrm{al}^{1}$ conducted a nationwide survey investigating the association between smartphone use and sleep among Japanese junior and high school students. They found that $84.4 \%$ of Japanese adolescents used smartphones
Correspondence: Taishiro Kishimoto Department of Psychiatry, Keio University School of Medicine, 35 Shinanomachi, Shinjuku, 160-8582, Tokyo, Japan

Email taishiro-k@mti.biglobe.ne.jp 
every day and that $8.3 \%$ of them used smartphones even after they turned off the room lights. In addition, they found that using smartphones after they turned off the room lights related to sleep disturbance.

Melatonin is a hormone produced by the pineal gland at night and under conditions of darkness in both diurnal and nocturnal species. Therefore, exposure to light during the night can affect the production of melatonin. In fact, especially the short-wavelength of blue lights (380-495 nm) at night is reported to retard or even cease the production of nocturnal melatonin. ${ }^{2-5}$ As melatonin plays an important role in controlling the night-day cycle, suppression of melatonin by light during the night has been implicated in the disruption of sleep. High-intensity displays, such as computers and smartphones, contain short wavelengths like a blue light. Therefore, the utilization of those displays could potentially have a negative impact on sleep.

In addition to the brightness of displays, viewing distance might play some role in lowering sleep quality. Bababekova et $\mathrm{al}^{6}$ examined whether the viewing distance of smartphones could alter asthenopia. They reported that the mean distance for text messaging and net surfing was shorter than a typical distance for viewing written material (36.2, 32.2, and $40 \mathrm{~cm}$, respectively). Furthermore, they found that the closer distance increased demands on both accommodation and vergence, which could exacerbate the symptoms of asthenopia. Although their study added valuable knowledge to the literature, they did not investigate the influence of viewing distance on sleep. Regarding the viewing distance, we have investigated people's body position when viewing smartphones elsewhere. ${ }^{7}$ Based on our survey targeting 138 nursing students, we found that they used smartphones almost every night before sleep, even after they turned off the lights. The time spent in the sitting position was shorter than the lying position $(P<0.05)$, and among those in the lying position, they favored the lateral lying position rather than the dorsal and/or prone positions $(P<0.01)$.

Given that exposure to blue lights can have a negative impact on sleep quality and that the viewing distance may modify the magnitude of this impact, we sought to determine the relationship between the smartphone viewing distance and subjective sleep status (eg, sleep quality, sleep efficiency, and sleep latency). We first measured the smartphone viewing distances utilizing an infrared depth sensor. Next, we investigated the severity of sleep disturbance using multiple rating scales and looked for the relationship between the viewing distance and sleep disturbance. We hypothesized that a closer viewing distance would have a greater negative impact on sleep.

\section{Participants and methods}

All participants provided their written consent before enrollment in the study. The study was approved by the Institutional Review Board of Aino University Junior College. This study was conducted according to the principles of the Declaration of Helsinki. All participants were compensated for this study.

\section{Participants}

Twenty-three nursing students (male 4/23, mean age \pm $\mathrm{SD}=19.7 \pm 3.1$ years) participated in the study. We recruited healthy volunteers, but a systematic examination of psychiatric or physical diseases that can alter sleep was not conducted. All participants owned a smartphone and reported that they use it before sleep.

\section{Assessment of subjective sleep status}

Subjective sleep status was assessed using the Pittsburgh Sleep Quality Index (PSQI), ${ }^{8,9}$ morningness-eveningness questionnaire (MEQ), ${ }^{10,11}$ and the Epworth sleepiness scale (ESS). ${ }^{12-15}$

\section{Determination of the viewing distance}

Microsoft Kinect $\subset$ for Windows was used for motion capture in order to determine participants' viewing distance of smartphones. We used the distance between the head and the hand while holding a smartphone to measure the viewing distance. This distance was calculated using the three-dimensional (3D) coordinates obtained by Kinect, a noncontact motionsensing device. The Kinect sensor acquires depth information by measuring reflection patterns with an infrared camera sensor. Using the Kinect analysis software, we can capture images and track and recognize the human body. Several studies have shown that Kinect is a useful measurement tool for distance, as it provides accurate information with an error of only a few millimeters. ${ }^{16,17}$ We developed a custom program to measure viewing distances using Kinect. This program provides $3 \mathrm{D}$ coordinates of any body part which we can specify using the Kinect's color images and skeleton data. Details of the materials used in this experiment are listed in Table 1. We considered that the bias would be minimal with this device because traditional motion capture systems require invasive sensors or electrical cords.

\section{Experimental conditions}

We measured viewing distances while using smartphones in sitting and lying positions for all subjects. We had participants hold their phones for 14.5 minutes in sitting position and 22.3 minutes in lying position because in a previous study 
Table I Materials used in the experiment

\begin{tabular}{ll}
\hline Microsoft Kinect $\odot$ for Windows & \\
Sensing range & $80-40 \mathrm{I} \mathrm{cm}$ \\
Horizontal direction & $57^{\circ}$ \\
Vertical direction & $43^{\circ}$ \\
Tilt motor & $\pm 27^{\circ}$ \\
Recording device & Windows 7 \\
Program environment & Microsoft Visual C++ 20I0 Express \\
& Kinect $\odot$ for Windows SDK v1.6 \\
& OpenCV 2.4.2 \\
Smartphone & iPhone 5 (Apple) \\
Maximal brightness & $500 \mathrm{~cd} / \mathrm{m}^{2}$ \\
\hline
\end{tabular}

we had conducted, people answered that they would use their phones for the respective minutes on average. ${ }^{7}$ The room lightness was $600 \mathrm{~lx}$ based on the eye position of participants. All participants used an Apple iPhone 5 with limited functionality to browse certain content with Google Chrome, but they could not check email, social network sites (SNSs) (such as Twitter and Facebook), or watch videos. They were all iPhone users and were all familiar with operating the phone. The luminance level of the smartphone display was fixed at $500 \mathrm{~cd} / \mathrm{m}^{2}$, and participants were not allowed to change the luminance level. In the laboratory room, the temperature was $25 \pm 5^{\circ} \mathrm{C}$ and the humidity was $50 \pm 5 \%$. Participants were asked to use the smartphone as they would normally use it. In the sitting position, participants sat in a chair (seat size: $360 \times 360 \mathrm{~cm}$ and height: $46 \mathrm{~cm}$ ) with a straight back without a desk. The Kinect was placed $180 \mathrm{~cm}$ vertically to the left side of the chair and $60 \mathrm{~cm}$ above the floor. In the lying position, subjects laid on a bed (bed size: $90 \times 200 \mathrm{~cm}$ and height: $40 \mathrm{~cm}$ ) in arbitrary lying positions (eg, dorsal position, prone position, and lateral position) to measure their viewing distance. We set up the Kinect $180 \mathrm{~cm}$ above the bed over the participants' chest area in order to avoid occlusions (Figure 1). The viewing distance was measured and recorded every 10 seconds by the Kinect for Windows SDK v1.6 program. The experiment was done between 1:30 pm and 5:30 pm. In order for participants to not be affected by the presence of the researcher in the room, a partition was placed between participant and researcher.

\section{Experimental procedures}

Participants were randomly assigned to start in either sitting position or lying position. Viewing distance in the sitting/ lying position was measured for the length of time mentioned before, which was followed by a 5-minute interval period, after which the viewing distance in the other position was measured. After the experiment, questionnaires were administered, which included demographic characteristics of the participants and a subjective sleep status using multiple rating scales, ie, PSQI, MEQ, and ESS.

\section{Illuminance measurement by multiple distances/angles}

As a separate experiment, we investigated the illuminance and spectrum for the light emitted from the smartphone display. In order to take into consideration the decrease in the illuminance in relation to the viewing distance, we measured at distances of 10,20, and $30 \mathrm{~cm}$. At each distance, we faced the smartphone forward 90,120 , and $150^{\circ}$. The screen image was set at the default Google search engine website in smartphones. The illuminance was measured three times at each distance and the mean was calculated. We measured the experiment room in a blackout situation for $0 \mathrm{~lx}$.

\section{Statistical analyses}

Differences between viewing distances in each body position were examined with a paired $t$-test. For the relationship between viewing distance and subjective sleep status, we used multiple regression analyses. As dependent variables, we used
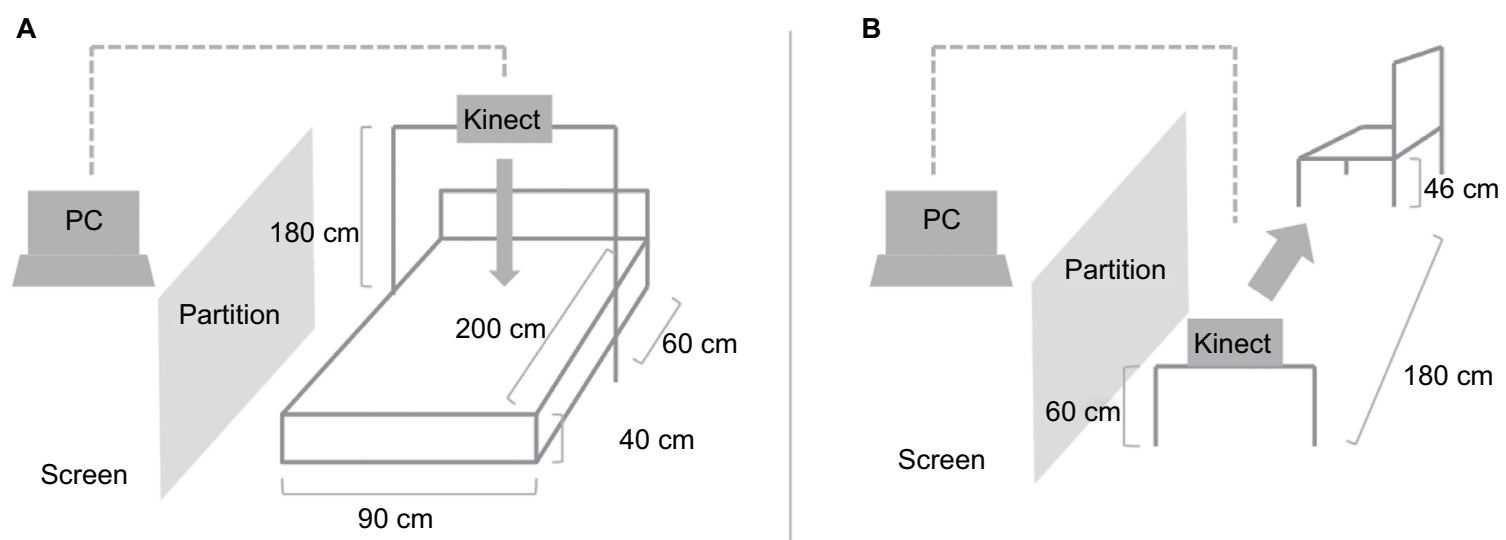

Figure I The layout for the experimental settings.

Notes: (A) layout for lying position. (B) Layout for sitting position. 
sleep state, sleep efficiency, sleep latency (determined from the PSQI score), circadian rhythm type (determined from the MEQ score), and daytime sleepiness (determined from the ESS score). As predictor variables, we included age, sex, visual acuity, the viewing distance in the sitting position, and the viewing distance in the lying position. All analyses were performed using SPSS 11.0J for Windows.

\section{Results}

\section{Sleep scale scores and viewing distances}

The mean \pm SD scores of PSQI, ESS, and MEQ were $5.82 \pm 1.76,10.95 \pm 3.59$, and 44.05 \pm 7.81 , respectively.

\section{The average viewing distances}

The smartphone viewing distance in the sitting position ranged from 13.3 to $32.9 \mathrm{~cm}$ among participants. In the lying position, it ranged from 9.9 to $21.3 \mathrm{~cm}$. The mean \pm $\mathrm{SD}$ of viewing distance was longer in the sitting position than in the lying position $(20.3 \pm 4.7$ vs $16.4 \pm 2.7$, respectively, $t(21)=3.604, P<0.01)$.

\section{The relationship between viewing distance and subjective sleep status}

Based on multiple regression analyses, we found that the short viewing distance in the lying position related positively to a poorer sleep state $\left(R^{2}=0.27, P<0.05\right)$, lower sleep efficiency $\left(R^{2}=0.35, P<0.05\right)$, and longer sleep latency $\left(R^{2}=0.38\right.$, $P<0.05)$. On the other hand, there were no significant associations between the sitting viewing distance and any of the sleep variables (Table 2).

\section{Illumination intensity of smartphones}

Within the spectrum of the smartphone light, the peak was at $453 \mathrm{~nm}$ (Figure 2). As shown in Figure 3, the illuminances decreased as the smartphone was moved farther away. Regarding the angle of the smartphone, the illuminance was the highest when the smartphone was directly facing the illuminometer $\left(90^{\circ}\right)$ as opposed to when it was placed at tilted positions $\left(120^{\circ}\right.$ and $\left.150^{\circ}\right)$. Based on the findings of the mean distances of smartphone viewing distances in sitting and lying positions and assuming that the viewing angle ranged from 90 to $120^{\circ}$, the calculated illuminance was from 25.3 to $42.6 \mathrm{~lx}$ when sitting and from 50.5 to $80.4 \mathrm{~lx}$ when lying.

\section{Discussion}

To the best of our knowledge, this is the first study investigating the viewing distance of smartphones before sleeping and the relationship with subjective sleep status. We found that the viewing distance in the lying position was shorter than that in the sitting position. Based on the findings of measured

Table 2 The relationship between the viewing distance and sleep determined by PSQI, MEQ, and ESS

\begin{tabular}{|c|c|c|c|c|c|c|c|c|c|c|}
\hline \multirow[t]{3}{*}{ Response variables } & \multicolumn{10}{|l|}{ PSQI } \\
\hline & \multicolumn{2}{|c|}{$\begin{array}{l}\text { Sleep quality (global } \\
\text { score) }\end{array}$} & \multicolumn{2}{|c|}{$\begin{array}{l}\text { Sleep } \\
\text { efficiency }\end{array}$} & \multicolumn{2}{|c|}{$\begin{array}{l}\text { Sleep } \\
\text { latency }\end{array}$} & \multicolumn{2}{|l|}{ MEQ } & \multicolumn{2}{|l|}{ ESS } \\
\hline & $a$ & $b$ & $a$ & $b$ & $a$ & $b$ & $a$ & $b$ & $a$ & $b$ \\
\hline Viewing distance in sitting position & -0.03 & -0.03 & 0.03 & 0.04 & -0.03 & -0.03 & 0.05 & 0.23 & 0.36 & 0.44 \\
\hline Viewing distance in lying position & -0.53 & $-0.47^{*}$ & -0.62 & $-0.55^{*}$ & -0.58 & $-0.54^{*}$ & -0.36 & -0.12 & -0.25 & -0.18 \\
\hline Age & -0.10 & -0.11 & -0.22 & -0.24 & -0.24 & -0.21 & -0.82 & -0.34 & 0.38 & 0.33 \\
\hline Sex & 0.04 & 0.04 & -0.01 & -0.01 & 0.10 & 0.08 & 7.15 & 0.36 & -2.10 & -0.23 \\
\hline Eyesight & 0.12 & 0.12 & 0.24 & 0.25 & 0.25 & 0.21 & 8.47 & 0.42 & -4.36 & -0.46 \\
\hline$R^{2}$ & 0.27 & & 0.35 & & 0.38 & & 0.36 & & 0.52 & \\
\hline
\end{tabular}

Notes: $a$ : partial regression coefficient. $b$ : standard regression coefficient. $* P<0.05$.

Abbreviations: ESS, Epworth sleepiness scale; MEQ, morningness-eveningness questionnaire; PSQI, Pittsburgh Sleep Quality Index.

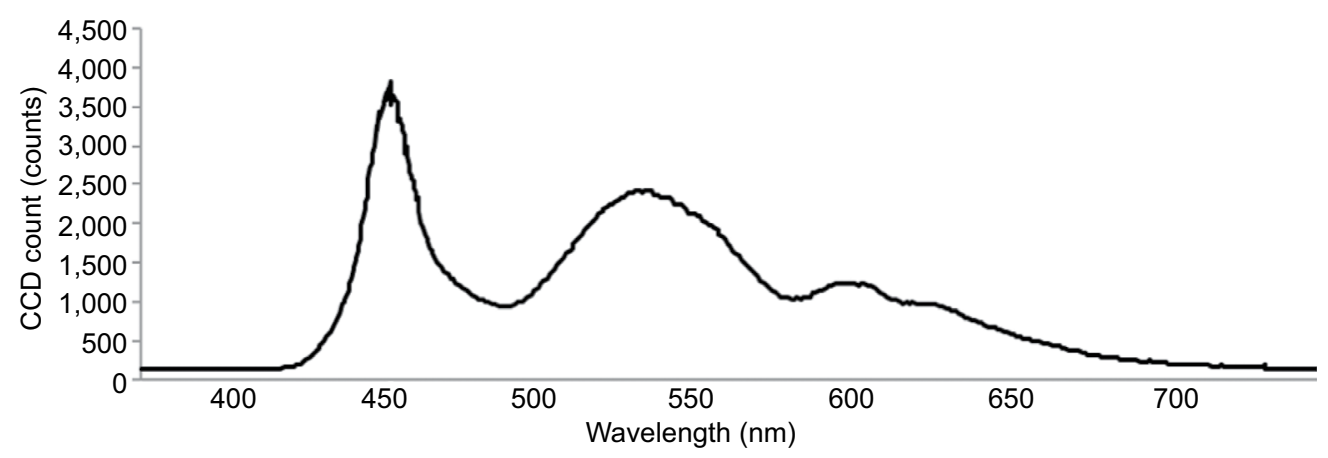

Figure 2 Spectral radiometric profile of the smartphone (measured at $90 \% 10 \mathrm{~cm}$ from iPhone 5). Abbreviation: CCD, Charge- Coupled Device. 


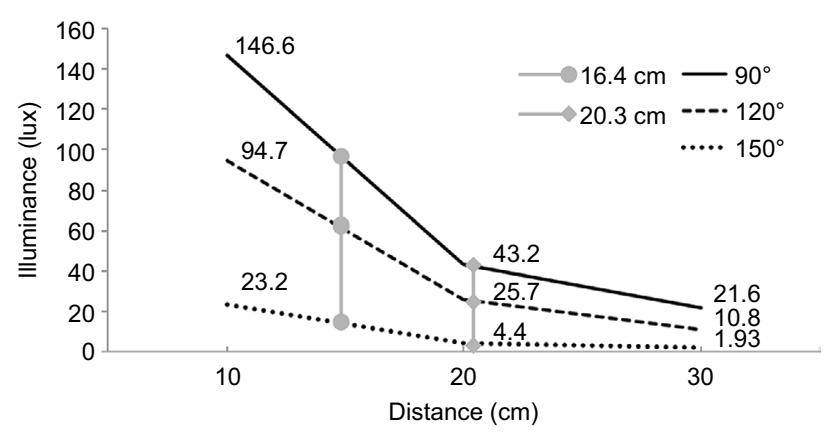

Figure 3 Illuminance of smartphone at various distances and angles. Notes: Gray circles and diamonds show the illuminances at the mean distances measured in this experiment.

illuminances from smartphones placed at multiple distances, it was estimated that students were exposed to 50.5-80.4 lx of lights, with the peak spectrum being $\sim 453 \mathrm{~nm}$. Moreover, based on a multiple regression analysis, only the distance of smartphones in the lying position predicted a poorer sleep state $\left(R^{2}=0.27, P<0.05\right)$, lower sleep efficiency $\left(R^{2}=0.35\right.$, $P<0.05)$, and longer sleep latency $\left(R^{2}=0.38, P<0.05\right)$ as assessed by PSQI. These findings suggest that people are exposed to light through smartphone monitors in high illuminance that mainly consists of blue light. It is of note that the viewing distance also correlated negatively with the subjective sleep status. Intrinsically photosensitive retinal ganglion cells (ipRGCs) express photopigment melanopsin, and ipRGCs project to several brain nuclei, including the suprachiasmatic nucleus (SCN) ${ }^{18}$ The SCN sends information to the pineal gland to modulate body temperature and produce hormones such as cortisol and melatonin. ${ }^{19-21}$ Because melanopsin photoreceptors reach peak light absorption at blue light wavelengths $\sim 480 \mathrm{~nm}$, it is thought that blue light affects the circadian rhythms. ${ }^{18}$

In 1996, Boivin et $\mathrm{al}^{22}$ reported that even low intensity light $(\sim 180$ lx) can affect circadian rhythm based on the body temperature measurement, whereas it had been thought that bright light $(\sim 7000-13000$ lx) was needed to synchronize circadian rhythm in humans. Boivin and Czeisler ${ }^{23}$ confirmed the findings by showing the effect of light on melatonin suppression in 1998. In 2000, Brainard et $\mathrm{al}^{24}$ reported that the spectrum of $446-477 \mathrm{~nm}$ was the most potent wavelength region in providing circadian input for the regulation of melatonin secretion. Thapan et $\mathrm{a}^{25}$ also confirmed this finding. They reported that a spectrum of 457-462 nm suppressed melatonin secretion the most. Since then, there has been increasing evidential support for the theory that blue light even when the illuminance is low - can affect sleep. Lockley et $\mathrm{al}^{26}$ compared $460 \mathrm{~nm} 5 \mathrm{~lx}$ light and $555 \mathrm{~nm} 68.1 \mathrm{~lx}$ light and found that the former light quality suppressed melatonin more. Warman et $\mathrm{al}^{27}$ showed that short-wavelength light $(\sim 460 \mathrm{~nm})$ even as low as $8 \mathrm{~lx}$ induced a melatonin phase shift that was seen when exposed to 12000 lx white light.

Since smartphone monitors emit blue light, there are multiple studies that have investigated the negative impact of smartphones on sleep. Chang et $\mathrm{al}^{3}$ reported that evening use of an e-Reader phase delays the circadian clock and suppresses melatonin secretion. They conducted a randomized crossover study with 12 healthy adults to compare the impact of light-emitting devices with a printed book, on sleep. They found that light-emitting devices suppressed evening melatonin levels by $55.12 \pm 20.12 \%$, whereas the printed book did not $(-18.77 \pm 98.57 \%)$. Moreover, based on polysomnography (PSG) and the Karolinska sleepiness scale, light-emitting devices decreased rapid eye movement (REM) sleep and decreased sleepiness in the evening. Figueiro et al ${ }^{4}$ investigated the impact of light emitted from computer monitors. When participants were exposed to $40 \mathrm{~lx}$ short-wavelength light from blue light-emitting goggles while watching a computer monitor, their melatonin levels were significantly reduced. When participants were exposed only to the computer monitors, the melatonin reduction was not as great as when wearing goggles, but a reduction was still seen compared to when they viewed the monitors through orange-tinted glasses. Wood et $\mathrm{al}^{5}$ conducted a similar study that investigated the impact of the light emitted from tablet displays, such as iPad2s. They reported that participants' melatonin levels decreased when they were exposed to the tablet's light as compared to the control conditions, although the effect was not as strong as when they were exposed to tablets and blue light-emitting goggles.

Although these experiments enriched our knowledge of how smartphones and/or tablets can affect sleep, these experiments were mainly conducted in an experimental environment and may not predict real-world usage of these devices. Most of the studies described earlier fixed the distance of the smartphone to $30-50 \mathrm{~cm}$, whereas our study showed that the average viewing distance in the real-world was $20.3 \mathrm{~cm}$ when sitting and $16.4 \mathrm{~cm}$ when lying. These statistics were determined based on markerless motion capture techniques where the subjects were not affected by experimental equipment and were able to exhibit their natural behavior.

The results of this study need to be interpreted in the context of the following limitations. First, we did not measure the duration of participants' smartphone usage, which can affect sleep quality given the findings of previous studies. We only recruited students who use smartphones before sleeping every night; however, the duration of use may be different day-to-day. Because we investigated the participants' sleep quality with rating scales, which assess the examinee's 
subjective sleep in the past month, it would not be practical to take into consideration a duration of usage that could fluctuate every day. Relating to this, the rating scales that were used in the study assess not the state, but rather trait or usual sleep habits of the subjective sleep status of the past month. In this study, it was impossible to examine the direct influence of the viewing distance on the subject's quality of sleep; rather, this study is suited to examining the relationship between normal smartphone-viewing habits and subjective sleep quality. Second, we did not measure melatonin levels, electroencephalogram (EEG), and/or other biological indices. The focus of this study was to investigate the viewing distance of smartphones and its impact on subjective sleep status in daily life, and trying to take such measurements would have disrupted the participants' natural usage of smartphones which we were attempting to observe. Third, we prepared bedding such as sheets and comforters for the research, and this unfamiliar bedding could have affected the participants' body position, as opposed to how they would sit/lay in their own beds. Moreover, we measured the distance only in two body positions - namely sitting and lying - but some people may prefer other positions. In addition, we set the room illuminance as $600 \mathrm{~lx}$ in the experiment. The brightness of each participant's room at home will differ, which might have some influence on the viewing distance. Fourth, we did not rule out potential psychiatric and/or physical diseases that can alter sleep. Although we recruited healthy volunteers for this study, some of them might have had such diseases and could have biased the results of the study. Finally, we conducted this study in the daytime.

\section{Conclusion}

We investigated the viewing distance of smartphones by using markerless motion capture techniques. We found that the distances were shorter in the lying position than in the sitting position, and altogether, these distances were shorter than those observed in previous studies. Moreover, smartphone distances correlated negatively with subjective sleep status. Therefore, when recommending ideal smartphone use, one should take into account the viewing distances.

\section{Acknowledgment}

The authors thank Mr Tsukasa Sugiura for his very helpful suggestions about programming the Kinect.

\section{Disclosure}

Taishiro Kishimoto has received consultant fees from Dainippon Sumitomo, Novartis, and Otsuka and has received speaker's honoraria from Banyu, Eli Lilly, Dainippon
Sumitomo, Janssen, Novartis, Otsuka, and Pfizer, outside the submitted work. He has also received grant support from the Pfizer Health Research, Takeda, Tanabe-Mitsubishi, Dainippon-Sumitomo, Otsuka, and Mochida, outside the submitted work. Masaru Mimura has received grants and/ or speaker's honoraria from Abbvie, Asahi Kasei, Astellas, Chugai, Cracie, Daiichi Sankyo, Dainippon-Sumitomo, Eisai, Eli Lilly, Fuji Film, Janssen, Meiji Pharma, Mochida, MSD, Novartis, Ono, Otsuka, Pfizer, Shionogi, Takeda, and Yoshitomi Pharmaceutical within the past 3 years, outside the submitted work. Kazuo Tsubota has received grants from Jins Co., Ltd, Toshiba Materials, and Tsubota Lav, outside the submitted work; in addition, he has a patent Tsubota Lab., Inc., pending and a patent Jins Co., Ltd., pending. The authors report no other conflicts of interest in this work.

\section{References}

1. Munezawa T, Kaneita Y, Osaki Y, et al. The association between use of smartphones after lights out and sleep disturbances among Japanese adolescents: a nationwide cross-sectional survey. Sleep. 2011; 34(8):1013-1020.

2. Cajochen C, Frey S, Anders D, et al. Evening exposure to a light-emitting diode (LED)-backlit computer screen affects circadian physiology and cognitive performance. J Appl Physiol. 2011;110(5):1432-1438.

3. Chang AM, Aeschbach D, Duffy JF, Czeisler CA. Evening use of lightemitting eReaders negatively affects sleep, circadian timing, and nextmorning alertness. Proc Natl Acad Sci U S A. 2014;112(4):1232-1237.

4. Figueiro MG, Wood B, Plitnick B, Rea MS. The impact of light from computer monitors on melatonin levels in college students. Neuro Endocrinol Lett. 2011;32(2):158-163.

5. Wood B, Rea MS, Plitnick B, Figueiro MG. Light level and duration of exposure determine the impact of self-luminous tablets on melatonin suppression. Appl Ergon. 2013;44(2):237-240.

6. Bababekova Y, Rosenfield M, Hue JE, Huang RR. Font size and viewing distance of handheld smartphones. Optom Vis Sci. 2011;88(7):795-797.

7. Yoshimura M, Kitazawa M, Kishimoto T, Mimura M, Tsubota K. A survey of Japanese young adults' postures when using smartphones before sleeping. J Mobile Technol Med. 2016;5(2):51-53.

8. Buysse DJ, Reynolds CF, Monk TH, Berman SR, Kupfer DJ. The Pittsburgh Sleep Quality Index: a new instrument for psychiatric practice and research. Psychiatry Res. 1989;28(2):193-213.

9. Doi Y, Minowa M, Uchiyama M, et al. Psychometric assessment of subjective sleep quality using the Japanese version of the Pittsburgh Sleep Quality Index (PSQI-J) in psychiatric disordered and control subjects. Psychiatry Res. 2000;97:165-172.

10. Horne JA, Östberg O. A self-assessment questionnaire to determine morningness-eveningness in human circadian rhythms. Int J Chronobiol. 1976;4:97-110.

11. Ishihara K, Miyashita A, Inugami M, Fukuda K, Yamazaki K, Miyata Y. The results of investigation by the Japanese version of MorningnessEveningness Questionnaire. Shinrigaku Kenkyu. 1986;57(2):87-91.

12. Johns MW. A new method for measuring daytime sleepiness: the Epworth sleepiness scale. Sleep. 1991;14(6):540-545.

13. Johns MW. Reliability and factor analysis of the Epworth sleepiness scale. Sleep. 1992;15(4):376-381.

14. Johns M, Hocking B. Daytime sleepiness and sleep habits of Australian workers. Sleep. 1997;20(10):844-849.

15. Takegami M, Suzukamo Y, Wakita T, et al. Development of a Japanese version of the Epworth Sleepiness Scale (JESS) based on item response theory. Sleep Med. 2009;10(5):556-565.

16. Khoshelham K, Elberink SO. Accuracy and resolution of Kinect depth data for indoor mapping applications. Sensors (Basel). 2012;12(2):1437-1454. 
17. Kurillo G, Han JJ, Obdržálek S, et al. Upper extremity reachable workspace evaluation with Kinect. Stud Health Technol Inform. 2013;184: 247-253.

18. Lucas RJ, Peirson SN, Berson DM, et al. Measuring and using light in the melanopsin age. Trends Neurosci. 2014;37(1):1-9.

19. Altun A, Ugur-Altun B. Melatonin: therapeutic and clinical utilization. Int J Clin Pract. 2007;61(5):835-845.

20. Kräuchi K, Cajochen C, Werth E, Wirz-Justice A. Functional link between distal vasodilation and sleep-onset latency? Am J Physiol Regul Integr Comp Physiol. 2000;278(3):R741-R748.

21. Saper CB, Lu J, Chou TC, Gooley J. The hypothalamic integrator for circadian rhythms. Trends Neurosci. 2005;28(3):152-157.

22. Boivin DB, Duffy JF, Kronauer RE, Czeisler CA. Dose-response relationships for resetting of human circadian clock by light. Nature. 1996;379(6565):540-542.
23. Boivin DB, Czeisler CA. Resetting of circadian melatonin and cortisol rhythms in humans by ordinary room light. Neuroreport. 1998;9(5): 779-782.

24. Brainard GC, Rollag MD, Hanifin JP, Van den Beld G, Sanford B. The effect of polarized versus nonpolarized light on melatonin regulation in humans. Photochem Photobiol. 2000;71(6):766-770.

25. Thapan K, Arendt J, Skene DJ. An action spectrum for melatonin suppression: evidence for a novel non-rod, non-cone photoreceptor system in humans. $J$ Physiol. 2001;535(pt 1):261-267.

26. Lockley SW, Brainard GC, Czeisler CA. High sensitivity of the human circadian melatonin rhythm to resetting by short wavelength light. J Clin Endocrinol Metab. 2003;88(9):4502-4505.

27. Warman VL, Dijk DJ, Warman GR, Arendt J, Skene DJ. Phase advancing human circadian rhythms with short wavelength light. Neurosci Lett. 2003;342:37-40.
Nature and Science of Sleep

\section{Publish your work in this journal}

Nature and Science of Sleep is an international, peer-reviewed, open access journal covering all aspects of sleep science and sleep medicine, including the neurophysiology and functions of sleep, the genetics of sleep, sleep and society, biological rhythms, dreaming, sleep disorders and therapy, and strategies to optimize healthy sleep. The manuscript

\section{Dovepress}

management system is completely online and includes a very quick and fair peer-review system, which is all easy to use. Visit http://www. dovepress.com/testimonials.php to read real quotes from published authors. 